\title{
Soy Phosphatidylglycerol Reduces Inflammation in a Contact Irritant Ear Edema Mouse Model In Vivo
}

\author{
Ding Xie, Vivek Choudhary, Mutsa Seremwe, John G. Edwards, Angela Wang, \\ Aaron C. Emmons, Katherine A. Bollag, Maribeth H. Johnson, and Wendy B. Bollag \\ Charlie Norwood VA Medical Center, Augusta, Georgia (V.C., W.B.B.); Institute of Molecular Medicine and Genetics, Department \\ of Medicine (D.X., M.S., W.B.B.), Department of Physiology (D.X., V.C., M.S., A.W., A.C.E., K.A.B., W.B.B.), Department of Family \\ Medicine (D.X.), Department of Neuroscience and Regenerative Medicine (M.H.J.), and Division of Dermatology, Department of \\ Medicine (W.B.B.), Medical College of Georgia, Augusta University, Augusta, Georgia; and Apeliotus Technologies, Inc., \\ Philadelphia, Pennsylvania (J.G.E., W.B.B.)
}

Received August 26, 2017; accepted April 17, 2018

\begin{abstract}
We have previously shown that phosphatidylglycerol (PG) regulates the function of keratinocytes, the predominant cells that compose the epidermis, inhibiting the proliferation of rapidly dividing keratinocytes. In particular, soy PG, a PG mixture with a high proportion of polyunsaturated fatty acids, is efficacious at inhibiting these proliferating keratinocytes. Psoriasis is a skin disorder characterized by hyperproliferation of keratinocytes and inflammation. Data in the lung suggest that $P G$ in pulmonary surfactant inhibits inflammation. To investigate the possibility of using PG containing polyunsaturated fatty acids for the treatment of psoriasis, we examined the effect of soy PG on inflammation induced by the application of 12-O-tetradecanoylphorbol 13-acetate (TPA), a contact irritant, to mouse ears in vivo. We monitored ear thickness and weight
\end{abstract}

as a measure of ear edema, as well as CD45-positive immune cell infiltration. Our results indicate that soy PG when applied together with 1,25-dihydroxyvitamin $D_{3}$ (vitamin $D$ ), an agent known to acutely disrupt the skin barrier, suppressed ear edema and inhibited the infiltration of CD45-positive immune cells. On the other hand, neither PG nor vitamin D alone was effective. The combination also decreased tumor necrosis factor- $\alpha$ (TNF $\alpha)$ levels. This result suggested the possibility that $P G$ was not permeating the skin barrier efficiently. Therefore, in a further study we applied $P G$ in a penetrationenhancing vehicle and found that it inhibited inflammation induced by the phorbol ester and decreased CD45-positive immune cell infiltration. Our results suggest the possibility of using soy $P G$ as a topical treatment option for psoriasis.

\section{Introduction}

Keratinocytes undergo a precisely regulated pattern of proliferation and differentiation that is essential for proper formation of the epidermis as a physical and water-permeability barrier (Yuspa et al., 1990; Goldsmith, 1991). Defects in the regulation of this growth program result in an abnormal barrier and a variety of skin diseases, such as psoriasis (Langley, 2005). Psoriasis is characterized by hyperproliferation and abnormal differentiation of epidermal keratinocytes as well as inflammation, and it results in a reduced quality of life similar to that observed in patients with life-threatening illness (Rapp et al., 1999; Stern et al., 2004).

Our previous studies have suggested that the lipid second messenger phosphatidylglycerol (PG) can be formed by a

This work was supported in part by the National Institutes of Health National Institute of Arthritis and Musculoskeletal and Skin Diseases (Grant R41 AR055022), and a VA Research Career Scientist Award (to W.B.B.). The contents of this article do not represent the views of the Department of Veterans Affairs or the United States Government.

https://doi.org/10.1124/jpet.117.244756. signaling module composed of the glycerol channel, aquaporin3 (AQP3), and phospholipase D2 (PLD2) (Zheng and Bollinger Bollag, 2003; Bollag et al., 2007; Xie et al., 2014), which are colocalized in epidermal keratinocytes (Zheng and Bollinger Bollag, 2003). Phospholipase D (PLD) is a lipid-metabolizing enzyme that can catalyze both phospholipid hydrolysis to produce phosphatidate and a transphosphatidylation reaction using primary alcohols to generate phosphatidyl alcohols.

We have previously found that both in vitro and in intact keratinocytes PLD can convert glycerol, a physiologic alcohol, to $P G$ and that PG levels increase upon stimulation of keratinocytes with a differentiating agent, elevated extracellular calcium levels (Zheng et al., 2003). PG production is maximal (Zheng et al., 2003) at a calcium concentration that is optimal for triggering early differentiation (Yuspa et al., 1989), suggesting a potential role in this process. Furthermore, manipulating this novel AQP3/PLD2 signaling module alters keratinocyte differentiation (Bollag et al., 2007; Choudhary et al., 2015). Importantly, egg PG inhibits proliferation of rapidly dividing keratinocytes whereas in slowly dividing cells egg PG stimulates proliferation; however, the

ABBREVIATIONS: ANOVA, analysis of variance; AQP3, aquaporin-3; CD45, leukocyte common antigen; DOPG, dioleoylphosphatidylglycerol; PG, phosphatidylglycerol; PLD, phospholipase D; PLD2, phospholipase D2; TNF $\alpha$, tumor necrosis factor- $\alpha$; TPA, 12-O-tetradecanoyl-phorbol 13-acetate; vitamin D, 1,25-dihydroxyvitamin $\mathrm{D}_{3}$. 
related phospholipid phosphatidylpropanol has no effect (Bollag et al., 2007).

A subsequent study from our laboratory demonstrated that PG species possessing polyunsaturated fatty acids are effective at inhibiting keratinocyte proliferation. In contrast, PG species containing saturated or monounsaturated fatty acids stimulate the growth of slowly dividing keratinocytes (Xie et al., 2014). Soy PG, which contains a large percentage of polyunsaturated fatty acids, is particularly effective at inhibiting keratinocyte proliferation (Xie et al., 2014), suggesting its possible use as a treatment to suppress the keratinocyte hyperproliferation observed in psoriasis.

Psoriasis is also characterized by immune cell infiltration into the skin and inflammation, and it has been proposed that psoriasis is an immune-mediated skin disease (reviewed in Helwa et al., 2013). However, recent data have suggested that there is a complex interplay between keratinocytes and immune cells, with keratinocytes producing cytokines that recruit and activate immune cells, which secrete cytokines that further stimulate keratinocytes, establishing a vicious cycle of inflammation (reviewed in Sabat and Wolk, 2011; Brotas et al., 2012; Lowes et al., 2013). The importance of keratinocytes to skin lesion development can be observed in a transgenic mouse model in which c-Jun and JunB are deleted only in epidermal keratinocytes (under the control of the keratin 14 promoter). These mice exhibit psoriasiform lesions that persist in conditional double knockout mice lacking a fully functional immune system (also deficient in Rag2 or TNFR1), indicating that $\mathrm{T}$ cells, although important, are not the sole mediators of the observed skin phenotype (Zenz et al., 2005).

$\mathrm{PG}$, which is produced by alveolar cells as a significant component of pulmonary surfactant, has been shown to suppress inflammation induced by microorganisms and microbial products in the lung (Kuronuma et al., 2009). Furthermore, PG inhibits infection of airway epithelial cells by respiratory syncytial virus and influenza $\mathrm{A}$ in vitro and protects the lungs from the deleterious inflammatory effects of these viruses in vivo (Numata et al., 2010, 2012). These results suggest the possibility that PG may possess not only antiproliferative but also anti-inflammatory actions. To test whether PG is anti-inflammatory in skin, we used soy PG in a contact irritant mouse ear edema model to determine whether PG can inhibit skin inflammation in vivo. Our results indicate that PG can inhibit inflammation in vivo and suggest that it might do so in part by suppressing $\mathrm{TNF} \alpha$ levels in the skin.

\section{Materials and Methods}

In Vivo Contact Irritant Ear Edema Mouse Model. Our experiments were performed as described in Clark et al. (2014). Briefly, the ears of three to five male ICR CD-1 outbred mice (25-30 g; 5 to 6 weeks of age from Harlan Laboratories, Indianapolis, IN) were treated with acetone or 12-O-tetradecanoylphorbol 13-acetate (TPA; $0.03 \%$ in acetone). At 1 and 4 hours after TPA administration, the appropriate vehicle or the treatment in vehicle was applied to each ear. Ear thickness was measured using a caliper both before and at approximately 18-20 hours after TPA treatment before sacrifice.

After sacrifice, a circular ear punch biopsy $\left(4 \mathrm{~mm}^{2}\right)$ was taken, weighed, and fixed in formalin. Histologic evaluation included immunohistochemical staining for CD45 to quantify the number of infiltrating immune cells as well as for $\mathrm{TNF} \alpha$ immunoreactivity. All procedures were approved by the Institutional Animal Care and Use
Committee and were conducted in accordance with the Guide for the Care and Use of Laboratory Animals as adopted and promulgated by the U.S. National Institutes of Health.

Immunohistochemical and Immunofluorescent Staining. Sections $(10 \mu \mathrm{m})$ were cut from formalin-fixed paraffin-embedded ear biopsy samples and deparaffinized and rehydrated as described in Voss et al. (2011). After antigen retrieval, inhibition of endogenous peroxidase with hydrogen peroxide, and blocking of nonspecific antibody binding, the sections were incubated with anti-CD45 antibody (BD Pharmingen, Franklin Lakes, NJ) or anti-TNF $\alpha$ antibody (Novus Biologicals, Littleton, CO) and visualized with diaminobenzidine (brown staining) with counterstaining using hematoxylin (blue staining in figures). Alternatively, after incubating with an anti-TNF $\alpha$ antibody (Abcam, Cambridge, MA), the staining was visualized using a Cy3-conjugated secondary antibody.

All staining, except for a portion (right ear immunoreactivity) of the analysis shown in Fig. 6 for CD45-positive cells, was performed by Georgia Pathology Research Services (Augusta, GA) using standard protocols. Multiple random sections (four to eight per mouse separately from the left and right ear) were counted by two independent observers in a blinded fashion, counts averaged to determine a value for each mouse ear, and the values statistically analyzed. TNF $\alpha$ immunofluorescence was determined using ImageJ (National Institutes of Health, Bethesda, MD) analysis of random fields in four sections per mouse (from the left ear) and was quantified in terms of fluorescent intensity per area (in arbitrary units) in the demarcated epidermis.

Statistical Analyses. For all experiments illustrating cumulative data, values for each animal are shown as individual symbols, with a line indicating the mean value for each group. Values from the left and right ears of each mouse were averaged. Rank transformations of the data were used to stabilize variances and account for outlying observations.

For the experiment testing the effect of $\mathrm{PG}$ and vitamin $\mathrm{D}$ alone and in combination, a $2 \times 2$ analysis of variance (ANOVA) was performed and the interaction was tested. For the experiment with the penetration-enhancing vehicle, one-way ANOVA was used. Tukey's test was used to adjust for the post hoc multiple comparisons of the mean ranks for significant effects from all analyses. SAS 9.4 (SAS Institute, Cary, NC) was used for all analyses, and statistical significance was determined using a type I error of $5 \%$.

\section{Results}

Effects of Soy PG, Alone and in Combination with 1,25Dihydroxyvitamin $D_{3}$, on TPA-Induced Ear Edema. Our previous results (Bollag et al., 2007; Xie et al., 2014) suggested the possibility that $\mathrm{PG}$, in particular soy $\mathrm{PG}$, might be useful to inhibit keratinocyte proliferation in skin diseases, such as psoriasis, which are characterized by hyperproliferation. However, there is no widely accepted animal model of psoriasis that reproduces all of the hallmarks of this disease (reviewed in Danilenko, 2008). On the other hand, inflammation is a key aspect of psoriasis and can be induced by the application of the contact irritant TPA to mouse ears (Sheu et al., 2002; Fowler et al., 2003; Sur et al., 2008). Therefore, we used the TPA-induced ear edema model to examine the ability of PG and 1,25-dihydroxyvitamin $\mathrm{D}_{3}$ (vitamin $\mathrm{D}$ ) to decrease phorbol ester-elicited ear edema.

Vitamin D was included because of concern about the potential ability of $\mathrm{PG}$, which has a molecular mass of approximately $750 \mathrm{Da}$, to penetrate the permeability barrier of the skin, which tends to exclude agents with molecular masses greater than $600 \mathrm{Da}$ (Pathan and Setty, 2009). Acute exposure to vitamin $\mathrm{D}$ is known to disrupt the epidermal barrier (von Brenken et al., 1997). In addition, vitamin D 
has been used successfully to treat psoriasis in humans (Samarasekera et al., 2013). After measuring the initial thickness, mouse ears were treated with TPA in acetone $(0.03 \%$ weight:volume) or acetone alone at time 0 . At 1 and 4 hours later, ice-cold $95 \%$ ethanol/5\% water as the vehicle, or the vehicle containing soy $\mathrm{PG}$, vitamin $\mathrm{D}$, or the combination, was applied to each ear. Approximately 20 hours later, the mice were sacrificed, ear thickness measured, and a 4-mm punch biopsy was obtained, weighed, and fixed in formalin.

Figure 1A shows the change in ear thickness (from time 0) in the ears treated with the indicated agents. TPA clearly increased ear thickness relative to the untreated control by more than $0.3 \mathrm{~mm}$. (The lack of change in the ear thickness in the untreated control demonstrated the reproducibility of the measurement.) Neither soy PG alone nor vitamin D alone had any statistically significant effect on the increase in ear thickness induced by TPA. However, the combination of soy PG and vitamin D significantly reduced (by approximately 40\%) the change in ear thickness observed upon TPA exposure. Similar effects were observed in terms of the weights of the 4-mm punch biopsy samples, with TPA inducing a significant increase of almost $5 \mathrm{mg}$ and the combination of PG and vitamin D suppressing the TPAinduced change in ear weight by about $50 \%$ (Fig. 1B).

We also performed an immunohistochemical analysis of the ear biopsy samples, staining for CD45 (leukocyte common antigen), which is specifically expressed by hematopoietic cells other than erythrocytes and plasma cells and is thus a marker of immune cells. This procedure allows the determination of the effect of treatment on immune cell infiltration into the ear. Random fields from multiple sections were then photographed, and the number of CD45-positive cells were counted in a blinded fashion.

As shown in Fig. 2, TPA treatment significantly increased the number of immune cells infiltrating the treated ear by approximately 5-fold, and PG alone slightly but significantly reduced this increase (by about 25\%). Vitamin D alone had no significant effect on the number of CD45-positive infiltrating immune cells, but the combination produced a statistically significant inhibition of about $90 \%$ in the number of CD45-positive immune cells infiltrating the ear in response to TPA, returning this parameter to a value that was not statistically different from the control.

Finally, we used immunohistochemical analysis to examine $\mathrm{TNF} \alpha$ immunoreactivity in the skin. We elected to focus on this particular cytokine based on the ability of anti-TNF $\alpha$ reagents to successfully treat psoriasis (Brotas et al., 2012). As shown in Fig. 3, TPA induced a striking increase in $\mathrm{TNF} \alpha$ staining that was not dramatically affected by either PG or vitamin $\mathrm{D}$ alone. However, treatment with both $\mathrm{PG}$ and vitamin $\mathrm{D}$ greatly reduced $\mathrm{TNF} \alpha$ staining, returning it to a level not substantially different from the control.

Because immunohistochemical analysis is difficult to quantify, we performed further quantitative immunofluorescence analysis of the TNF $\alpha$ levels in additional sections from each mouse. Fluorescent staining of the epidermis was quantified in four sections per mouse; the results are presented in Fig. 4, with each symbol representing an individual mouse and the lines showing the mean values. TPA caused an approximate doubling of TNF $\alpha$ in the epidermis, with no significant effect of PG observed. On the other hand, vitamin D induced a significant decrease in $\mathrm{TNF} \alpha$ immunofluorescence that was not further affected by the concomitant addition of PG, returning both groups to a value that was not significantly different from the control. The disparity observed between Figs. 3 and 4 in terms of effects of vitamin D alone likely reflects the different techniques used for visualization; thus, enzyme (horseradish peroxidase)-based immunohistochemical analysis amplifies the signal and may accentuate lowintensity staining relative to immunofluorescence.

Effects of Different Concentrations of Soy PG in a Penetration-Enhancing Vehicle on TPA-Induced Ear Edema. Although vitamin D has been used successfully as a therapy for psoriasis, the acute effect of vitamin D treatment on mouse skin is a disruption of the epidermal permeability
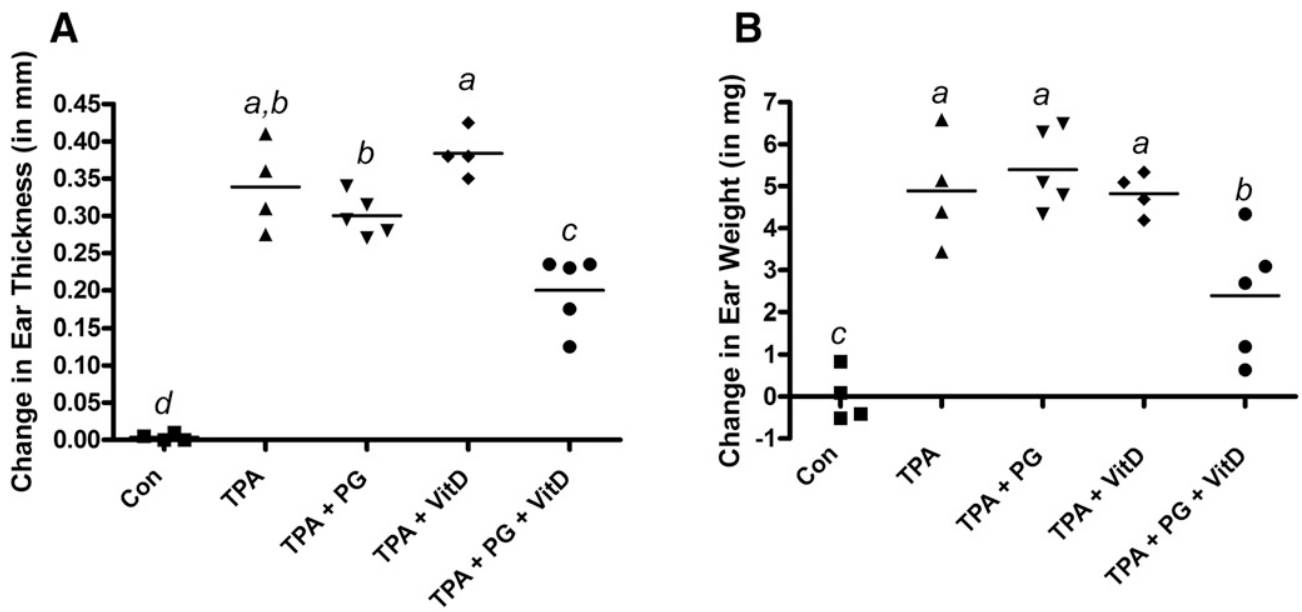

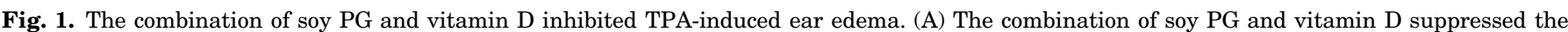

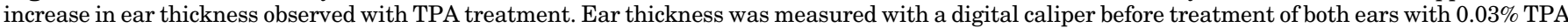

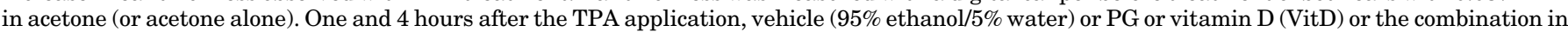

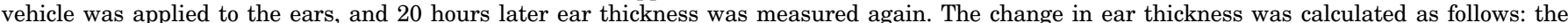

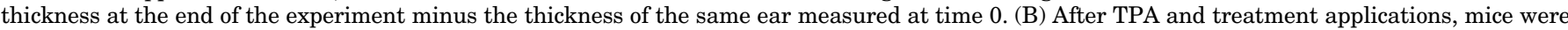

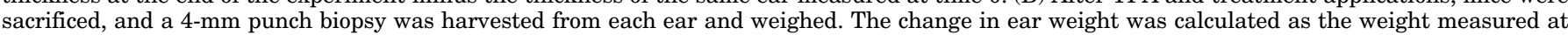

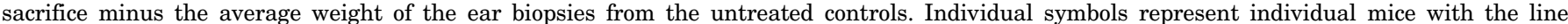

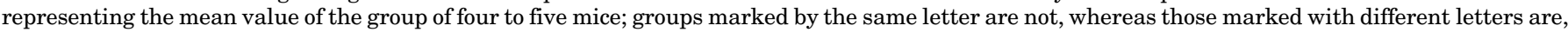

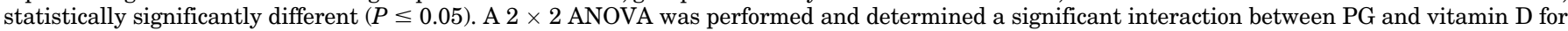
both change in thickness $(P=0.0021)$ and change in weight $(P=0.039)$. 

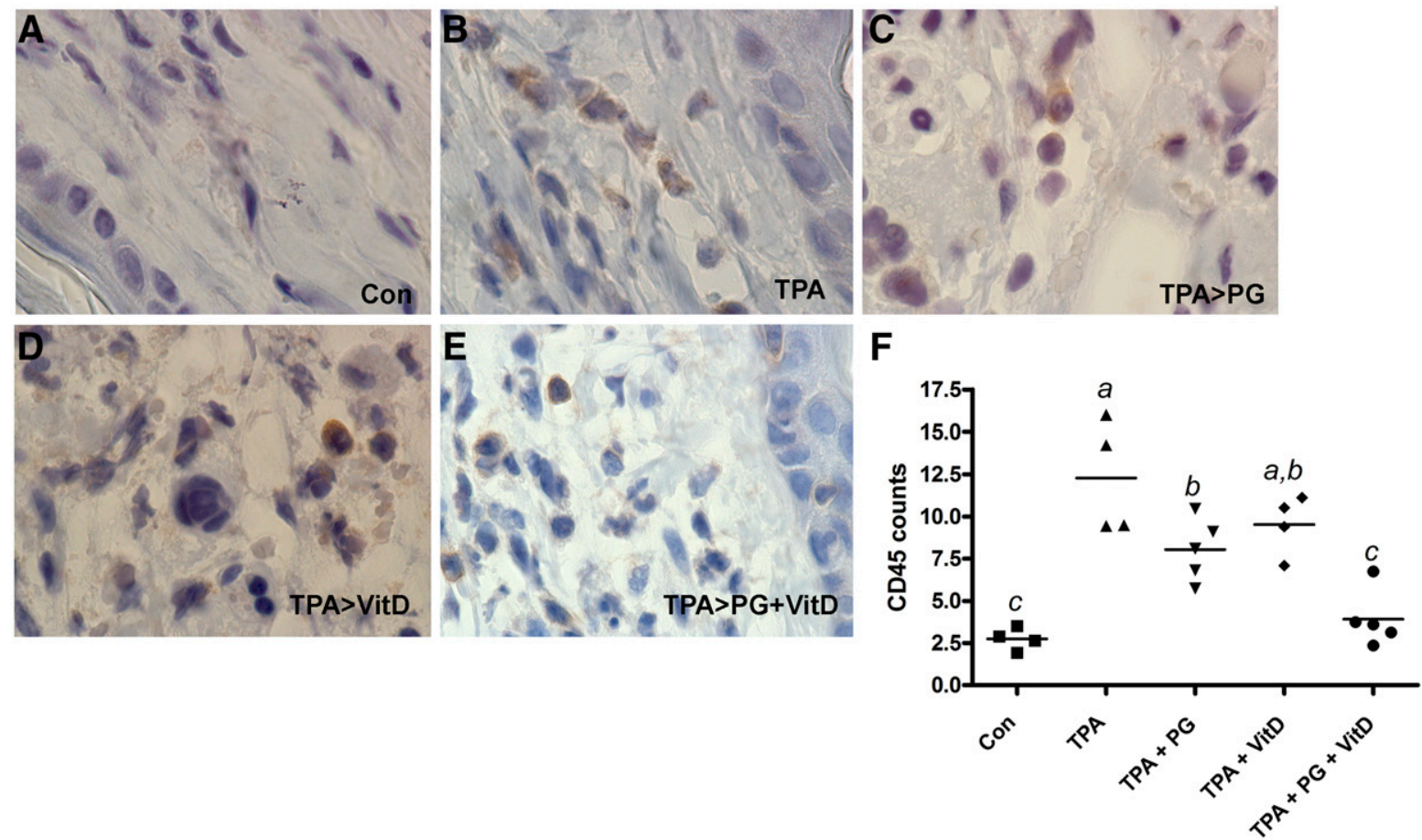

Fig. 2. The combination of soy PG and vitamin D suppressed TPA-induced immune cell infiltration into the ear. Multiple sections were cut from formalin-fixed, paraffin-embedded ear biopsies obtained from mice treated as in Fig. 1 and stained for CD45 as described in Materials and Methods. (A-E) Representative sections from (A) control mice and mice treated with (B) TPA, (C) TPA and PG, (D) TPA and vitamin D (VitD), and (E) TPA, PG, and VitD. (F) The number of CD45-positive cells was determined by counting immunostained (brown) cells in at least five random fields from a minimum of two sections from the left and right ear biopsies of treated mice. Cells were counted in a blinded manner by at least two independent observers, and the counts were averaged for each mouse. Mean values obtained from these counts for each mouse in a given treatment group were averaged and compared statistically as in Fig. 1. Individual symbols represent individual mice with the line representing the mean value of the group of four to five mice; groups marked by the same letter are not, whereas those marked with different letters are, statistically significantly different $(P \leq 0.05)$. The effect of each agent was found to be additive with a significant effect for both PG $(P=0.0001)$ and VitD $(P=0.012)$.

barrier (von Brenken et al., 1997). The ability of vitamin D, a barrier disruptor, to manifest an anti-inflammatory effect of PG in the absence of its own effects on inflammation suggested that permeation of the lipid through the skin could be an issue. We thus hypothesized that application of $P G$ in a vehicle that enhances permeability might improve the skin response to $\mathrm{PG}$ alone.

In consultation with Avanti Polar Lipids, we selected a vehicle for topical application composed of triacylglycerol, in particular, trioctanoin (8:0, 8:0, 8:0), and magnesium-stearate (18:0) at a ratio that yielded a cream with a consistency suitable for easy application. Again, we first measured the thickness of both ears of each mouse with a digital caliper (at time 0 ) followed by application of $0.03 \% \mathrm{TPA}$ (in acetone) to both ears. We then applied vehicle or vehicle containing $0.02 \%$ or $0.2 \%$ soy PG at 1 and 4 hours after TPA exposure.

At 18 hours after the TPA application, each mouse ear was again measured with the digital caliper, and the mice were
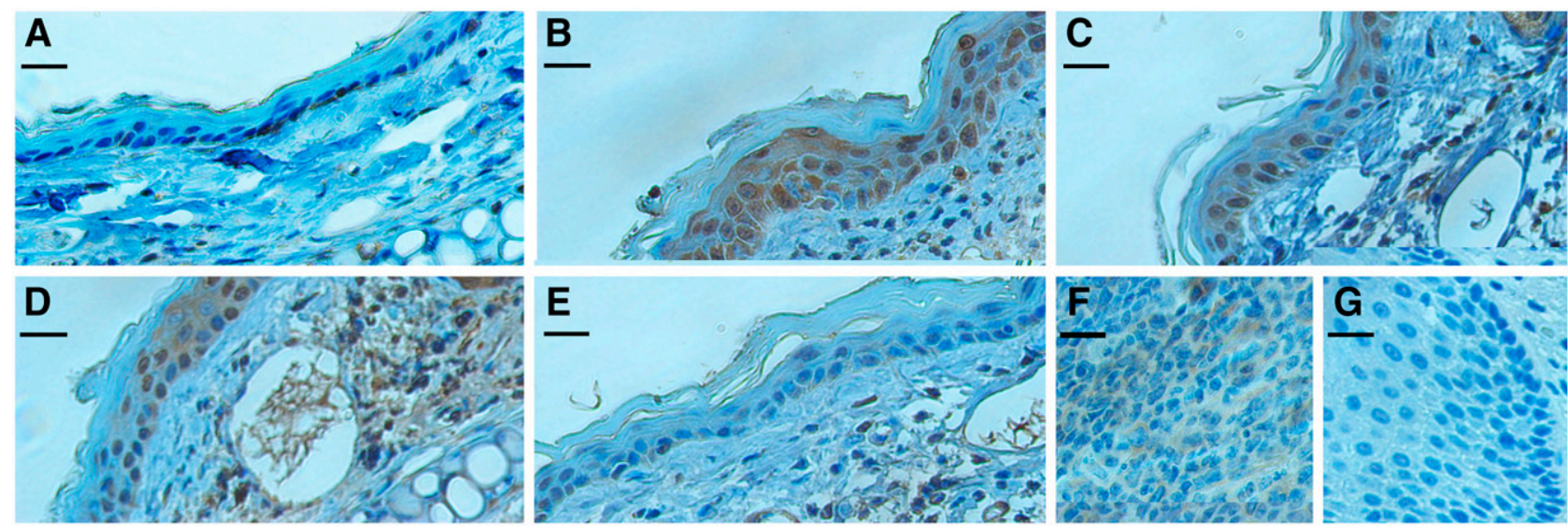

Fig. 3. The combination of soy PG and vitamin D suppressed TPA-induced TNF $\alpha$ levels. Multiple sections were cut from formalin-fixed, paraffinembedded ear biopsies obtained from mice treated as in Fig. 1 and stained for TNF $\alpha$ as described in Materials and Methods, with (A) control, (B) TPA, (C) TPA + PG, (D) TPA + vitamin D, (E) TPA + PG + vitamin D, (F) positive control (tonsil tissue), and (G) negative control (no primary antibody included). Results are representative of sections obtained from four to five mice. Scale bar, $20 \mu \mathrm{m}$. 


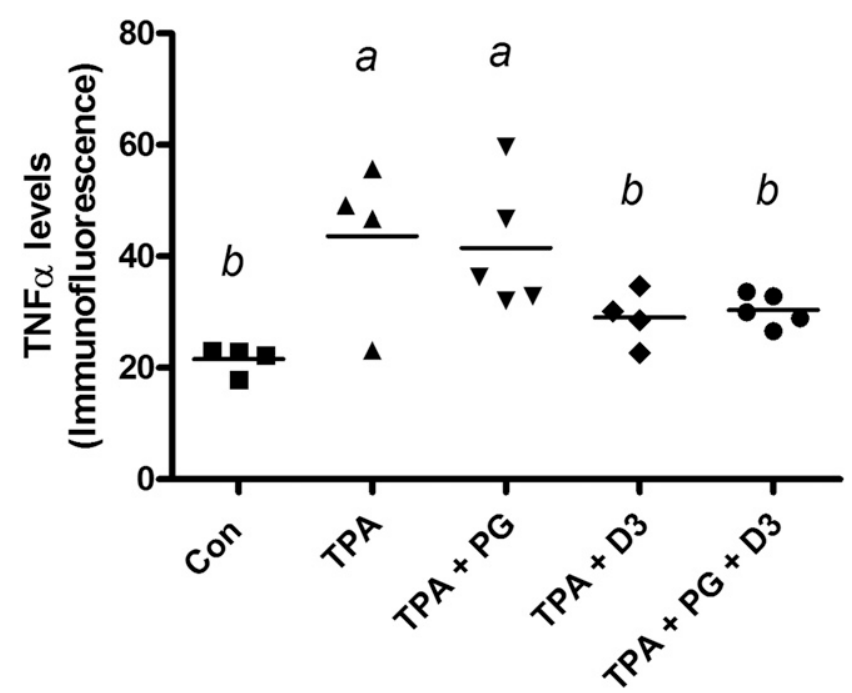

Fig. 4. TPA induced a significant increase in $\mathrm{TNF} \alpha$ levels, which were significantly reduced by vitamin D. Additional sections were cut from formalin-fixed, paraffin-embedded ear biopsies obtained from mice treated as in Fig. 3, stained for TNF $\alpha$ and immunofluorescence levels quantified using ImageJ as described in Materials and Methods. Results are representative of sections obtained from four to five mice. Individual symbols represent individual mice with the line representing the mean value of the group of four to five mice. A significant additive effect of vitamin $\mathrm{D}$ was observed $(P=$ 0.016 ); groups marked by the same letter are not, whereas those marked with different letters are, statistically significantly different $(P \leq 0.05)$.

sacrificed. Two punch biopsies were taken from each ear to measure weight and for immunohistochemical analysis. Because many vehicles themselves are known to exert effects in skin (Surber and Smith, 2005), an additional set of mice received TPA and a sham treatment (their ears were rubbed without applying vehicle or PG), providing a sham control to determine potential effects of the vehicle. A final set of mice received neither TPA stimulation nor treatment of any kind, providing a null control for TPA exposure and ear weight. The values were then calculated as the change in ear thickness (relative to time 0) or weight (relative to an average weight obtained from the ears of the null controls).

Similar to its effect in the previous experiment, TPA with the sham treatment resulted in an increase in ear thickness of approximately $0.3 \mathrm{~mm}$; however, by comparison the vehicle treatment statistically significantly reduced (by about 70\%) the TPA-induced increase in ear thickness (Fig. 5). Nevertheless, as illustrated in Fig. 5A, soy PG at both $0.02 \%$ and $0.2 \%$ applied topically in the trioctanoin vehicle was able to further reduce ear thickness by approximately $65 \%$ relative to the vehicle alone ( $n=4$ to 5 animals), with the low-dose PG appearing to be as effective as the higher dose in inhibiting inflammation. Again, there was essentially no change in ear thickness in the sham control. Similar results were obtained in terms of a reduction in ear weight (Fig. 5B), with the two PG doses returning the weight measure to a value that was not statistically significantly different from the control. Although the changes in weight values for the two PG doses were not statistically significantly different from the vehicle alone, this could be the result of the fact that each mouse could not be biopsied at time 0 to serve as its own control for this measurement.

Immunohistochemical analysis using an antibody to CD45 was also conducted, as previously noted. The results, shown in Fig. 6, indicate that TPA induced a statistically significant increase in the number of CD45-positive cells relative to unexposed ears. Treatment with vehicle did not significantly reduce the number of CD45-positive immune cells infiltrating the skin induced by TPA. However, PG treatment (analyzing the two concentrations of PG combined) resulted in a significant reduction in the number of infiltrating CD45-positive cells.

We also performed immunohistochemical analysis for $\mathrm{TNF} \alpha$ on ear biopsy sections from the treated mice. As shown in Fig. 7, TNF $\alpha$ staining was increased in the ears exposed to TPA alone (Fig. 7B) as compared with the control ears (Fig. 7A), and vehicle alone reduced TNF $\alpha$ immunoreactivity (Fig. 7C). There may have been a small additional effect of the vehicle
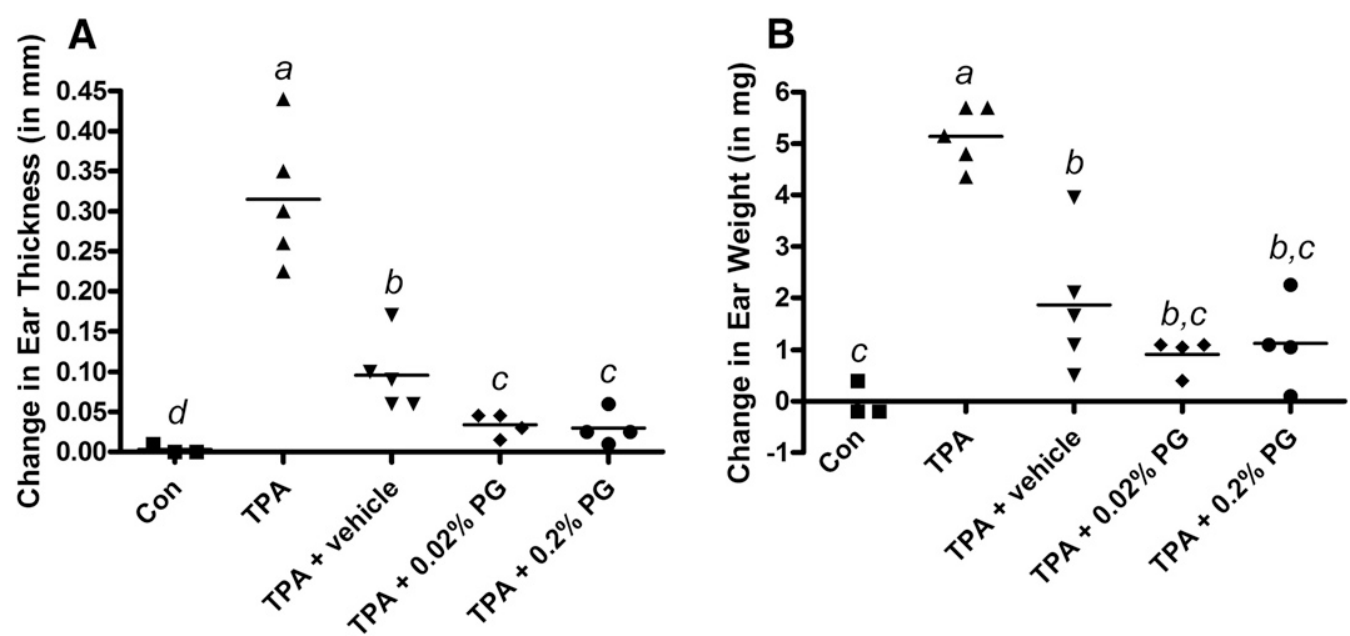

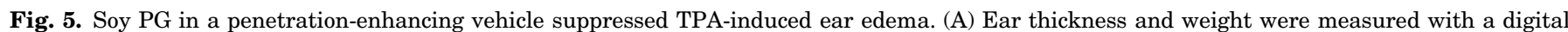

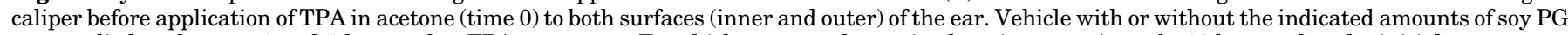

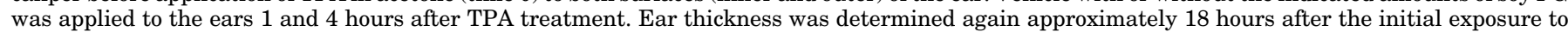

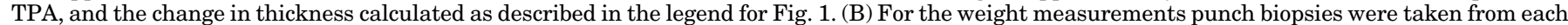

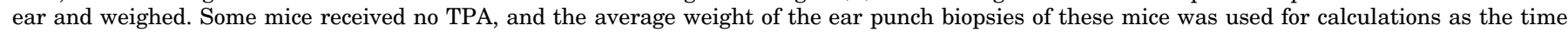

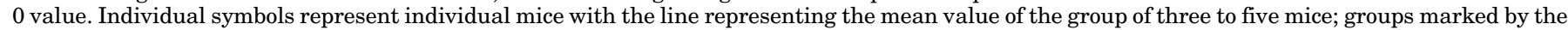
same letter are not, whereas those marked with different letters are, statistically significantly different $(P \leq 0.05)$. 

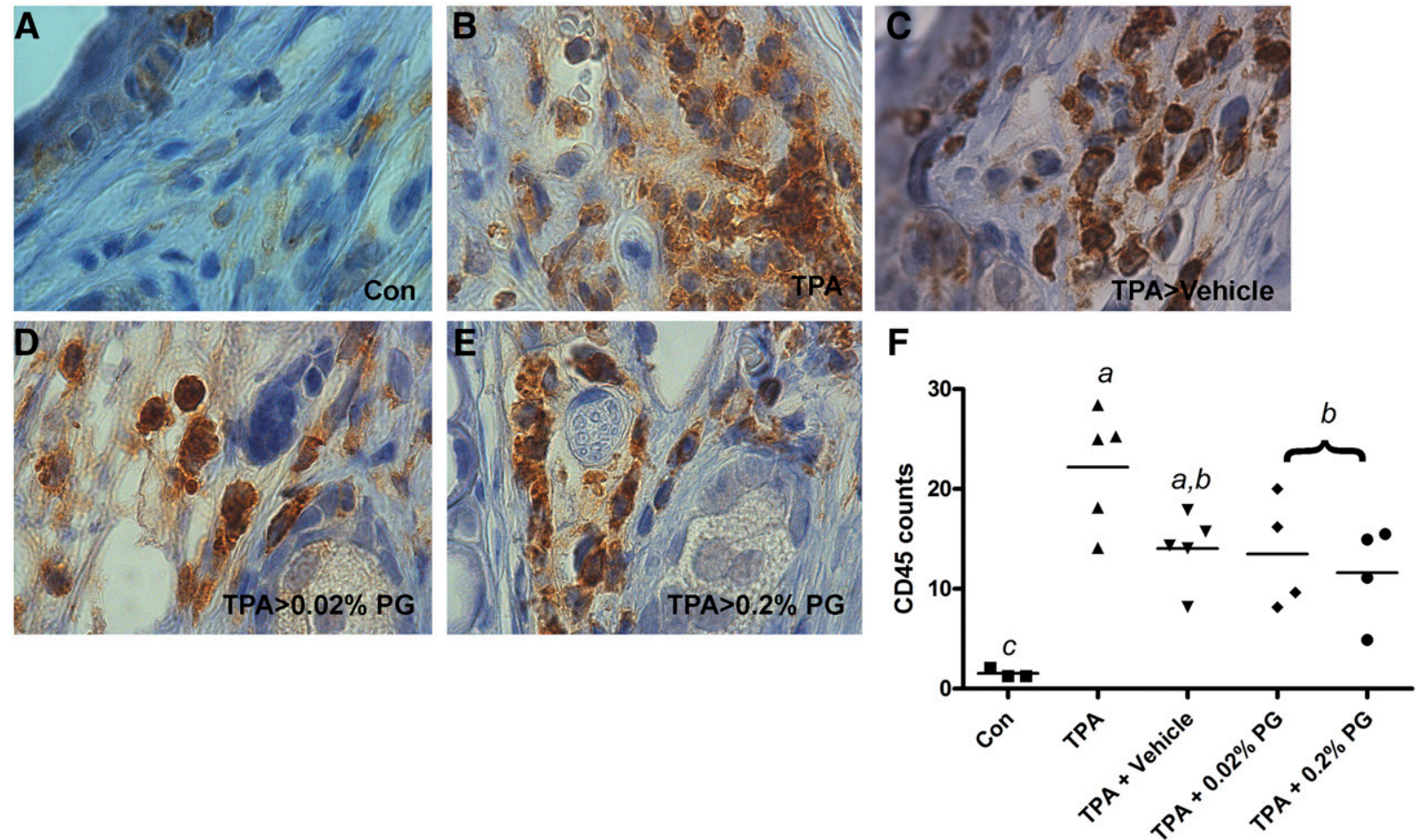

Fig. 6. Soy PG in a penetration-enhancing vehicle suppressed TPA-induced immune cell infiltration into the ear. Multiple sections were cut from formalin-fixed, paraffin-embedded ear biopsies obtained from mice treated as in Fig. 5 and stained for CD45 as described in Materials and Methods. (A-E) Representative sections from (A) control mice and mice treated with (B) TPA, (C) TPA and vehicle, (D) TPA and 0.02\% PG, and (E) TPA and 0.2\% PG. (F) The number of CD45-positive cells was determined by counting immunostained (brown) cells in at least five random fields from a minimum of two sections from the left and right ear biopsies of treated mice as in Fig. 5. Cells were counted in a blinded manner by at least two observers, and the counts were averaged for each mouse. Individual symbols represent individual mice with the line representing the mean value of the group of three to five mice; groups marked by the same letter are not, whereas those marked with different letters are, statistically significantly different $(P \leq 0.05)$.

containing the lower concentration of soy PG (Fig. 7D), although the higher PG dose did not appear to decrease $\mathrm{TNF} \alpha$ staining any more than the vehicle alone (Fig. 7E).

\section{Discussion}

Our previous study provided evidence for the existence in primary mouse keratinocytes of a novel lipid signaling pathway, for which PG is a key effector in the regulation of keratinocyte proliferation and differentiation. In particular, we showed that egg PG inhibits keratinocyte proliferation in rapidly dividing keratinocytes and stimulates keratinocyte proliferation in slowly dividing keratinocytes (Bollag et al., 2007). A further study suggested that PG species with different fatty acid compositions can exert different effects in keratinocytes (Xie et al., 2014). It is perhaps not surprising
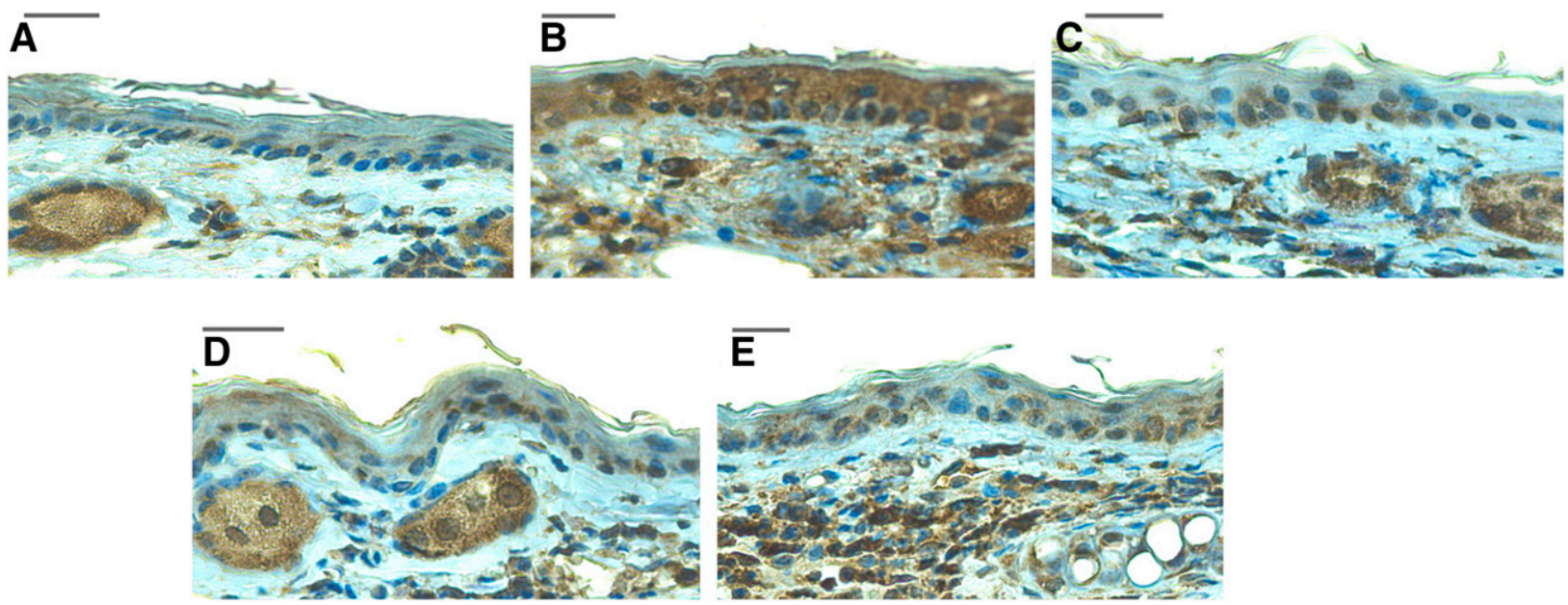

Fig. 7. Vehicle alone and vehicle containing soy PG suppressed TPA-induced TNF $\alpha$ levels. Multiple sections were cut from formalin-fixed, paraffinembedded ear biopsies obtained from mice treated as in Fig. 5 and stained for TNF $\alpha$ as described in Materials and Methods, with (A) control, (B) TPA, (C) TPA + vehicle, (D) TPA + vehicle containing $0.02 \%$ soy PG, and (E) TPA + vehicle containing $0.2 \%$ soy PG. Results are representative of sections obtained from three to five mice. Scale bar, $20 \mu \mathrm{m}$. 
that PG species with different acyl groups have different signaling functions, as in the lung PG species possessing saturated fatty acids cannot block the anti-inflammatory effects of surfactant protein A on lipopolysaccharide-treated macrophages while PG containing unsaturated fatty acids can (Chiba et al., 2006). Thus, our previous studies suggested that PG might be an ideal treatment to normalize skin function, with different PG species for different skin conditions.

Although in a previous study synthetic polyunsaturated fatty acid-containing PGs, and in particular dilinoleoylphosphatidylglycerol, seemed most effective at inhibiting keratinocyte proliferation in vitro (Xie et al., 2014), the expense of these PGs could potentially preclude their use as a treatment of psoriasis. Therefore, in this study we investigated the ability of soy PG, a mixture of PG species containing a high proportion of polyunsaturated fatty acids, as a potential treatment for psoriasis and the inflammation that accompanies this disease using a mouse model that mimics the inflammatory aspect of the disease. Soy PG also has the advantage of being a natural product.

When soy PG was applied in an ethanol/water vehicle, the lipid showed essentially no effect on ear edema (induced by TPA) by itself. However, in combination with vitamin $\mathrm{D}$, which alone also had no effect, soy PG inhibited the TPA-induced ear edema response. This result suggests the possibility of using soy $\mathrm{PG}$ in conjunction with vitamin $\mathrm{D}$, analogs of which are in current clinical use to treat psoriasis (Samarasekera et al. 2013), to more effectively control skin inflammation.

On the other hand, because vitamin $\mathrm{D}$ can disturb the epidermal barrier to allow better permeation of exogenous substances into mouse skin (von Brenken et al., 1997), this result suggested the possibility that soy $\mathrm{PG}$ alone was ineffective in penetrating the epidermis to exert its antiinflammatory effects but was able to do so when allowed entry through the vitamin D-disrupted barrier. Indeed, when applied in a penetration-enhancing cream vehicle, soy PG alone was able to inhibit TPA-induced ear edema.

However, it should be noted that in this experiment the vehicle itself was able to reduce inflammation and $\mathrm{TNF} \alpha$ immunoreactivity. Although the mechanism underlying the activity is unclear, an ability of vehicles to exert effects in the skin is often observed (Surber and Smith, 2005). For example, petrolatum can improve psoriasis (Limaye and Weightman, 1997); it is thought to do so through its ability to occlude the skin and reduce transepidermal water loss. Barrier disruption itself is reported to increase cytokine release (Wood et al., 1992) and contribute to psoriasis in mouse models (Roelandt et al., 2009; Nakajima et al., 2013) and patients (Roberson and Bowcock, 2010). Alternatively, the lipid-based vehicle may sequester the hydrophobic TPA and prevent its effective penetration and action in the skin.

TPA induces the expression of several inflammatory cytokines in keratinocytes (Carlsson et al., 2005; Helwa et al., 2015) and skin (Gebhardt et al., 2002; Mueller, 2006). These cytokines include $\mathrm{TNF} \alpha$, which is known to be elevated in psoriasis; in fact, drugs targeting the $\mathrm{TNF} \alpha$ pathway are effective in the treatment of psoriasis (Brotas et al., 2012). Indeed, our results showed that combined treatment with PG and vitamin D inhibited the TPA-induced increase in TNF $\alpha$ immunoreactivity in the skin. In separate immunofluorescent $\mathrm{TNF} \alpha$ staining, quantitation showed a significant inhibition by vitamin $\mathrm{D}$ alone. This result suggests that the effects of the combined treatment on $\mathrm{TNF} \alpha$ alone are not sufficient to explain the synergistic reduction in ear inflammation seen. Thus, in addition to inhibiting inflammation (PG) or promoting barrier disruption and reducing $\mathrm{TNF} \alpha$ levels (vitamin $\mathrm{D}$ ), these agents may have other actions that contribute to their synergistic effects in the ear edema model.

Some cytokines such as $\mathrm{TNF} \alpha$ are thought to originate from immune cells in psoriasis lesions; others, such as interleukin- $1 \alpha$, are thought to arise mainly from the keratinocytes (Brotas et al., 2012). Thus, it is unlikely that keratinocytes are the only or perhaps even the primary source of $\mathrm{TNF} \alpha$. On the other hand, as shown in Fig. 2, TPA induces immune cell infiltration that is not completely inhibited by either PG or vitamin D alone. Because this agent is also known to activate macrophages, among other immune cells, it seems likely that multiple cells contribute to the elevation in $\mathrm{TNF} \alpha$ levels in this model. Thus, it seems possible that cytokines produced either by immune cells or by keratinocytes can recruit and activate additional immune cells, thereby helping to initiate and/or maintain a cytokine network of inflammation and promote the development of psoriatic skin lesions. In this regard, the ability of 1,25-dihydroxyvitamin $\mathrm{D}_{3}$ to inhibit immune cell activation (Reichrath et al., 2007) may help to explain the observed inhibitory effect on epidermal $\mathrm{TNF} \alpha$ levels (Fig. 4).

In these experiments, we used male mice to demonstrate anti-inflammatory effects of soy PG. There is no apparent sex difference in the incidence psoriasis, although the severity of the disease is greater in men (Hagg et al., 2017), which is the reason for our initial decision to focus on males. However, it is critical that these anti-inflammatory effects of PG be investigated in females as well. Nevertheless, our experiments serve as proof of principle for the feasibility of potentially using PG as a therapy to treat psoriasis. Based on the positive effects observed in these experiments, we have elected to examine effects of PG in both sexes in a model that more accurately mimics the psoriatic phenotype rather than this acute inflammation contact irritant model. These experiments are currently in progress.

Data from Voelker and colleagues have demonstrated an ability of palmitoyl-oleoylphosphatidylglycerol (POPG) to inhibit inflammation in alveolar cells and the lung. Indeed, POPG effectively reduces microbial product-induced arachidonic acid release from human and mouse macrophages treated with Mycoplasma pneumonia membrane, without inhibiting cell surface binding of Mycoplasma (Kandasamy et al., 2011). These investigators have also shown that dioleoylphosphatidylglycerol (DOPG) can inhibit interleukin-8 production in BEAS2B human bronchial epithelial cells (Numata et al., 2013). In line with this result, Wu et al. (2003) also showed that DOPG effectively inhibits endotoxinstimulated type IIA secretory phospholipase $\mathrm{A}_{2}$ levels and activity via reductions in the activation of nuclear factor- $\kappa \mathrm{B}$ in macrophages. Our results show that soy PG also exerts antiinflammatory effects in the skin.

In conclusion, the present study shows for the first time to our knowledge that soy PG was able to suppress inflammation in response to a contact irritant (TPA) in an in vivo ear edema mouse model. This action may be related, at least in part, to the ability of soy PG to decrease the recruitment of immune cells, the infiltration of which is markedly increased in the inflammatory skin disease psoriasis. Thus, our results suggest 
that PG may be useful for treating skin diseases such as psoriasis, which are characterized by excessive keratinocyte proliferation and/or inflammation.

\section{Acknowledgments}

We thank Georgia Research Pathology Services (GRPS) for excellent technical assistance with immunohistochemistry and immunofluorescence and express particular appreciation to Kimya Jones of GRPS for her skilled and timely help.

\section{Authorship Contributions}

Participated in research design: Xie, Edwards, Bollag.

Conducted experiments: Xie, Seremwe.

Performed data analysis: Xie, Choudhary, Wang, Emmons, Bollag, Johnson, Bollag.

Wrote or contributed to the writing of the manuscript: Xie, Choudhary, Bollag.

\section{References}

Bollag WB, Xie D, Zheng X, and Zhong X (2007) A potential role for the phospholipase D2-aquaporin-3 signaling module in early keratinocyte differentiation: production of a phosphatidylglycerol signaling lipid. J Invest Dermatol 127:2823-2831.

Brotas AM, Cunha JM, Lago EH, Machado CC, and Carneiro SC (2012) Tumor necrosis factor-alpha and the cytokine network in psoriasis. An Bras Dermatol 87:673-681.

Carlsson H, Yhr M, Petersson S, Collins N, Polyak K, and Enerbäck C (2005) Psoriasin (S100A7) and calgranulin-B (S100A9) induction is dependent on reactive oxygen species and is downregulated by Bcl-2 and antioxidants. Cancer Biol Ther 4:998-1005.

Chiba H, Piboonpocanun S, Mitsuzawa H, Kuronuma K, Murphy RC, and Voelker DR (2006) Pulmonary surfactant proteins and lipids as modulators of inflammation and innate immunity. Respirology 11 (Suppl):S2-S6.

Choudhary V, Olala LO, Qin H, Helwa I, Pan ZQ, Tsai YY, Frohman MA, KaddourDjebbar I, and Bollag WB (2015) Aquaporin-3 re-expression induces differentiation in a phospholipase D2-dependent manner in aquaporin-3-knockout mouse keratinocytes. J Invest Dermatol 135:499-507.

Clark SP, Bollag WB, Westlund KN, Ma F, Falls G, Xie D, Johnson M, Isales CM, and Bhattacharyya MH (2014) Pine oil effects on chemical and thermal injury in mice and cultured mouse dorsal root ganglion neurons. Phytother Res 28:252-260.

Danilenko DM (2008) Review paper: preclinical models of psoriasis. Vet Pathol 45: 563-575.

Fowler AJ, Sheu MY, Schmuth M, Kao J, Fluhr JW, Rhein L, Collins JL, Willson TM, Mangelsdorf DJ, Elias PM, et al. (2003) Liver X receptor activators display antiinflammatory activity in irritant and allergic contact dermatitis models: liver$\mathrm{X}$-receptor-specific inhibition of inflammation and primary cytokine production. $J$ Invest Dermatol 120:246-255.

Gebhardt C, Breitenbach U, Tuckermann JP, Dittrich BT, Richter KH, and Angel P (2002) Calgranulins S100A8 and S100A9 are negatively regulated by glucocorticoids in a c-Fos-dependent manner and overexpressed throughout skin carcinogenesis. Oncogene 21:4266-4276.

Goldsmith LA (1991) Physiology, Biochemistry, and Molecular Biology of the Skin, Oxford University Press, New York.

Hägg D, Sundström A, Eriksson M, and Schmitt-Egenolf M (2017) Severity of psoriasis differs between men and women: a study of the clinical outcome measure psoriasis area and severity index (PASI) in 5438 Swedish register patients. Am $J$ Clin Dermatol 18:583-590.

Helwa I, Gulotto M, and Bollag WB (2013) Keratinocytes in psoriasis: key players in the disease process, in Psoriasis Types, Triggers and Treatment Strategies (Smith $\mathrm{P}$ and Johnson N eds) Nova Science, Hauppauge, NY

Helwa I, Patel R, Karempelis P, Kaddour-Djebbar I, Choudhary V, and Bollag WB (2015) The antipsoriatic agent monomethylfumarate has antiproliferative, prodifferentiative, and anti-inflammatory effects on keratinocytes. J Pharmacol Exp Ther 352:90-97.

Kandasamy P, Zarini S, Chan ED, Leslie CC, Murphy RC, and Voelker DR (2011) Pulmonary surfactant phosphatidylglycerol inhibits Mycoplasma pneumoniaestimulated eicosanoid production from human and mouse macrophages. J Biol Chem 286:7841-7853.

Kuronuma K, Mitsuzawa H, Takeda K, Nishitani C, Chan ED, Kuroki Y, Nakamura M, and Voelker DR (2009) Anionic pulmonary surfactant phospholipids inhibit inflammatory responses from alveolar macrophages and U937 cells by binding the lipopolysaccharideinteracting proteins CD14 and MD-2. J Biol Chem 284:25488-25500.

Langley R (2005) Psoriasis, Firefly Books, New York.

Limaye S and Weightman W (1997) Effect of an ointment containing boric acid, zinc oxide, starch and petrolatum on psoriasis. Australas J Dermatol 38:185-186.

Lowes MA, Russell CB, Martin DA, Towne JE, and Krueger JG (2013) The IL-23/T17 pathogenic axis in psoriasis is amplified by keratinocyte responses. Trends Immunol 34:174-181.
Mueller MM (2006) Inflammation in epithelial skin tumours: old stories and new ideas. Eur J Cancer 42:735-744.

Nakajima K, Terao M, Takaishi M, Kataoka S, Goto-Inoue N, Setou M, Horie K, Sakamoto F, Ito M, Azukizawa H, et al. (2013) Barrier abnormality due to ceramide deficiency leads to psoriasiform inflammation in a mouse model. J Invest Dermatol 133:2555-2565.

Numata M, Chu HW, Dakhama A, and Voelker DR (2010) Pulmonary surfactant phosphatidylglycerol inhibits respiratory syncytial virus-induced inflammation and infection. Proc Natl Acad Sci USA 107:320-325.

Numata M, Kandasamy P, Nagashima Y, Posey J, Hartshorn K, Woodland D, and Voelker DR (2012) Phosphatidylglycerol suppresses influenza A virus infection. Am J Respir Cell Mol Biol 46:479-487.

Numata M, Nagashima Y, Moore ML, Berry KZ, Chan M, Kandasamy P, Peebles RS, Jr, Murphy RC, and Voelker DR (2013) Phosphatidylglycerol provides short-term prophylaxis against respiratory syncytial virus infection. $J$ Lipid Res 54: 2133-2143.

Pathan IB and Setty CM (2009) Chemical penetration enhancers for transdermal drug delivery systems. Trop J Pharm Res 8:173-179.

Rapp SR, Feldman SR, Exum ML, Fleischer AB, Jr, and Reboussin DM (1999) Psoriasis causes as much disability as other major medical diseases. $J$ Am Acad Dermatol 41:401-407.

Reichrath J, Lehmann B, Carlberg C, Varani J, and Zouboulis CC (2007) Vitamins as hormones. Horm Metab Res 39:71-84.

Roberson ED and Bowcock AM (2010) Psoriasis genetics: breaking the barrier. Trends Genet 26:415-423.

Roelandt T, Giddelo C, Heughebaert C, Denecker G, Hupe M, Crumrine D, Kusuma A, Haftek M, Roseeuw D, Declercq W, et al. (2009) The "caveolae brake hypothesis" and the epidermal barrier. J Invest Dermatol 129:927-936.

Sabat R and Wolk K (2011) Research in practice: IL-22 and IL-20: significance for epithelial homeostasis and psoriasis pathogenesis. J Dtsch Dermatol Ges 9 518-523.

Samarasekera EJ, Sawyer L, Wonderling D, Tucker R, and Smith CH (2013) Topical therapies for the treatment of plaque psoriasis: systematic review and network meta-analyses. Br J Dermatol 168:954-967.

Sheu MY, Fowler AJ, Kao J, Schmuth M, Schoonjans K, Auwerx J, Fluhr JW, Man MQ, Elias PM, and Feingold KR (2002) Topical peroxisome proliferator activated receptor-alpha activators reduce inflammation in irritant and allergic contact dermatitis models. J Invest Dermatol 118:94-101.

Stern RS, Nijsten T, Feldman SR, Margolis DJ, and Rolstad T (2004) Psoriasis is common, carries a substantial burden even when not extensive, and is associated with widespread treatment dissatisfaction. J Investig Dermatol Symp Proc 9 136-139.

Sur R, Babad JM, Garay M, Liebel FT, and Southall MD (2008) Anti-inflammatory activity of sertaconazole nitrate is mediated via activation of a p38-COX-2-PGE2 pathway. J Invest Dermatol 128:336-344.

Surber C and Smith EW (2005) The mystical effects of dermatological vehicles. Dermatology 210:157-168.

von Brenken S, Jensen JM, Fartasch M, and Proksch E (1997) Topical vitamin D3 derivatives impair the epidermal permeability barrier in normal mouse skin. Dermatology 194:151-156.

Voss KE, Bollag RJ, Fussell N, By C, Sheehan DJ, and Bollag WB (2011) Abnormal aquaporin-3 protein expression in hyperproliferative skin disorders. Arch Dermatol Res 303:591-600.

Wood LC, Jackson SM, Elias PM, Grunfeld C, and Feingold KR (1992) Cutaneous barrier perturbation stimulates cytokine production in the epidermis of mice. $J$ Clin Invest 90:482-487.

Wu YZ, Medjane S, Chabot S, Kubrusly FS, Raw I, Chignard M, and Touqui L (2003) Surfactant protein-A and phosphatidylglycerol suppress type IIA phospholipase A2 synthesis via nuclear factor-kappaB. Am J Respir Crit Care Med 168:692-699.

Xie D, Seremwe M, Edwards JG, Podolsky R, and Bollag WB (2014) Distinct effects of different phosphatidylglycerol species on mouse keratinocyte proliferation. PLoS One 9:e107119.

Yuspa SH, Hennings H, Tucker RW, Kilkenny A, Lee E, Kruszewski F, and Roop DR (1990) The Regulation of Differentiation in Normal and Neoplastic Keratinocytes, Wiley-Liss, New York.

Yuspa SH, Kilkenny AE, Steinert PM, and Roop DR (1989) Expression of murine epidermal differentiation markers is tightly regulated by restricted extracellular calcium concentrations in vitro. J Cell Biol 109:1207-1217.

Zenz R, Eferl R, Kenner L, Florin L, Hummerich L, Mehic D, Scheuch H, Angel P, Tschachler E, and Wagner EF (2005) Psoriasis-like skin disease and arthritis caused by inducible epidermal deletion of Jun proteins. Nature 437:369-375.

Zheng X and Bollinger Bollag W (2003) Aquaporin 3 colocates with phospholipase d2 in caveolin-rich membrane microdomains and is downregulated upon keratinocyte differentiation. J Invest Dermatol 121:1487-1495.

Zheng X, Ray S, and Bollag WB (2003) Modulation of phospholipase D-mediated phosphatidylglycerol formation by differentiating agents in primary mouse epidermal keratinocytes. Biochim Biophys Acta 1643:25-36.

Address correspondence to: Dr. Wendy B. Bollag, Department of Physiology, Medical College of Georgia at Augusta University, 1120 15th Street, Augusta, GA 30912. E-mail: wbollag@augusta.edu 